\title{
12. Nerventransplantation: Überbrückung eines Defektes des Nervus ulnaris mit einem lyophilisierten und desantigenisierten homologen Transplantat
}

Film

\author{
W. JACOBY, R. FaHLBUSCH und B. MackerT-München
}

Nach Veröffentlichung von 2 erfolgreichen Transplantationen ${ }^{1}$ (Nervus ulnaris und Nervus medianus) mit homologen lyophilisierten Nerven Anfang des Jahres 1970 haben wir (bis 1. 3. 1971) bei 53 Patienten (die Ausfälle bestanden bis zu $11 \mathrm{Jah}$ ren) insgesamt 58 gleichartige Transplantationen bis $13 \mathrm{~cm}$ Länge durchgeführt, wobei in allen Fällen gleichzeitig eine Tubulisation mit lyophilisierter homologer Dura erfolgte. Es handelt sich um 13 Transplantationen am Nervus medianus, 16 am Nervus ulnaris, 6 am Nervus radialis, 1 am Nervus ischiadicus, 6 am Nervus peronäus, 1 am Nervus femoralis, 5 am Armplexus und um 10 in einer Sitzung durchgeführte Transplantationen an mehreren Nerven (meist Nervus ulnaris und medianus kombiniert). Bei keinem dieser Fälle traten postoperativ Unverträglichkeitsreaktionen bzw. Wundheilungsstörungen auf.

Außer den bereits veröffentlichten 2 erfolgreichen Transplantationen gelangten bisher weitere 15 Fälle zur Nachuntersuchung. Obwohl einige dieser Patienten die theoretisch zu errechnende Restitutionszeit (bei Zugrundelegung einer täglichen Restitutionsstrecke von $2 \mathrm{~mm}$ ) noch nicht erreicht haben, wurde bei allen klinisch und elektromyographisch bereits eine weitgehende Restitution registriert (Tab. 1). Die Beurteilung der Funktionsrückkehr ergab bei 14 nachuntersuchten Patienten ein gutes und bei 3 Patienten ein befriedigendes Ergebnis. Bei keinem der Patienten fehlte die Restitution.

Der Fỉm zeigt einen 25jährigen Patienten (Programmierer) mit einem kompletten Ulnaris-Ausfall nach 7 Monate vorher erfolgter Verletzung im distalen Unterarmdrittel rechts. Der präoperative klinische Befund am Patienten, der an seinem Arbeitsplatz nicht mehr eingesetzt werden konnte, wird demonstriert. Die bei der Operation am Nerven erhobenen Befunde und die angewendete Technik bei der Einfügung eines $50 \mathrm{~mm}$ langen Transplantates, sowie die dann durchgeführte Tubulisation mit lyophilisierter Dura werden gezeigt. Bereits 120 Tage nach der Operation war eine fast komplette motorische und sensible Restitution des Ulnaris-Ausfalles eingetreten. Nach Wiedererlangung seiner Arbeitsfähigkeit ist der Patient bei seiner manuellen Tätigkeit am Arbeitsplatz zu sehen.

\section{Vereinigung von Arterien mit klebenden Ringen}

\section{Farbfilm}

\section{R. GotTLoB und G. BLÜMeL-Wien/Österreich}

Gefäße mit einem äußeren Durchmesser von weniger als $2 \mathrm{~mm}$ lassen sich mit den derzeit verfügbaren Apparaten nicht vereinigen. Eine Anastomosierung ist nur bei Anwendung von Operationsmikroskopen technisch durchführbar. Das darge-

1 Jacoby, W., Fahlbusch, R., Mackert, B., Braun, B., Rolle, I., Schnell, I.: Utberbrückung peripherer Nervendefekte mit lyophilisierten und desantigenisierten homologen Transplantaten. Mehn. med. Wschr. 112, 586-589 (1970). 